\title{
Review on Learning based Interface Modelling using Augmented Reality
}

\author{
Akshay Indalkar ${ }^{1}$, Akshay Gunjal $^{2}$, Rachna Satao $^{3}$, Mihir Ashok Dalal ${ }^{4}$, Nikhil Sharma ${ }^{5}$ \\ Student, Computer Department, Smt. Kashibai Navale College of Engineering, Pune-411041, India ${ }^{1,2,4,5}$ \\ Assistant Professor, Computer Department, Smt. Kashibai Navale College of Engineering, Pune-411041, India ${ }^{3}$
}

\begin{abstract}
Augmented Reality (AR) is a widely growing field with a lot of scope in future. The learning aspects of AR have been widely studied from student's perception and learning effectiveness using AR. Making use of interactive concepts involving user involvement (via speech, actions, gestures) such as AR based User Interfaces(UIs) increases the efficiency of user learning. Augmented Reality can be used as learning element where user interacts with the developed augmented reality based Graphical user interface (GUI) to understand the different concepts which the user wants to learn. It helps the user for easy learning and better understanding of various concepts to be learned.
\end{abstract}

Keywords: Augmented Reality, Hand Gestures, Shader Effects, Unity, Vuforia

\section{INTRODUCTION}

Augmented Reality (AR) is an approach to create a 3dimentional virtual real world whose elements are augmented using computer generated sensory input. The sensory information which is put into it can be Graphics, sound, GPS or video [4] that is used to create a virtual real world. Augmented Reality changes the way we view the world. It is significantly a subfield of virtual reality. It combines virtual object with real world which is known as mediated reality.

Augmented Reality technology can be used to enhance the concentration and understanding of user.AR in learning [1] has been great impact on learning behaviour of student or user. It has been studied from the students perspective and learning efficiency which makes use of AR based learning model. In this case an Augmented Reality English learning system is used by embedding English lessons in it. This helps to understand the teachers and students perception. The perception is how students find it useful in learning perspective and how teachers find it useful in teaching perspective. After completing the AR based English learning system courses the students and teachers were asked to give the feedback of the AR learning courses.

The feedback was significantly interesting and justifies the use of AR based learning model which help in learning. Various feedback are as follows.

- It helped student to learn English lesson easily making it interesting and fun.

- AR learning model motivate the student for learning and better understand the contents.

- Students wanted to learn through AR in future.

- Its helps students to memorize the contents and able to concentrate more on studies.

- The AR English class was quite different from the monotonous teaching classes.
- Students hoped that they could use the AR at their home.

Teachers viewpoints upon the AR based learning is that it helped teachers for better explanation of a particular topic, Also increases the teachers professional skill for teaching. Lastly, teachers need not to prepare lot of study material only the 3-Dimentional GUI based AR model is sufficient. Another case of learning through AR based system which is a Picture Puzzle Education System [2] which is used to enhance the creativity, helps growth of small muscles and help mental processes of perception, reasoning, memory and judgement of children which is also known as Cognition along with English learning.AR based Picture Puzzle Education System contains different blocks (puzzles) which when combined together form a single picture and after that corresponding 3D model of the picture is displayed. The child needs to interact with the Puzzle system and needs to arrange the block (puzzles) to form 3D model which acts as a catalyst to boost the creativity of the children.Fig 1 describes various block and Fig. 2. describes the formed picture. Once the 3D model is generated the puzzle system generates English sound which describes the name of the 3D model. So naturally the children will also learn English through it.

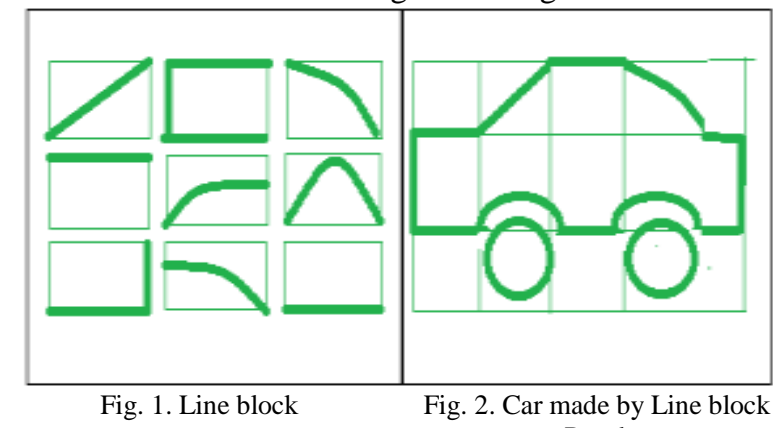
Puzzle 


\section{INTERACTION WITH AUGMENTED REALITY SYSTEM VIA HAND GESTURES}

Hand gestures can be defined as certain movements or actions done by the user's hands that may possibly depict a response or a form of communication media. Hand gestures allow user to be interactive with the provided AR based system interface. Thereby increasing the involvement in the system with an increased interest. Augmented reality and hand gestures can be combined together to yield interactivity which is easy to understand from user's perspective and also provides a way to immerse the user in the virtual environment to have a greater impact on the user. Now to detect a hand gesture, it consist of a sequence of steps that involves colour models, Image transformation, processing [3] and gesture detection. Basically a video recorder (or simply a camera) records the user's hand gestures. The recorded sequence of images (the video feed) is then processed to detect the stored hand gestures.

The gesture processing part consists of steps like image blurring, grey scale transformation, thresholding etc. The preliminary processing results in filtering out other image elements to locate the user's moving hands. Once the hands movement is filtered out, the type of gesture can be identified. Blobs are used to identify hand gesture in final detection stage. Blobs are black and white images where the moving part appears white. On recognising a gesture a database can be used to map command in the system by classifying the type of gesture stored in the database. Fig. 3 shows the hand gesture detection module.

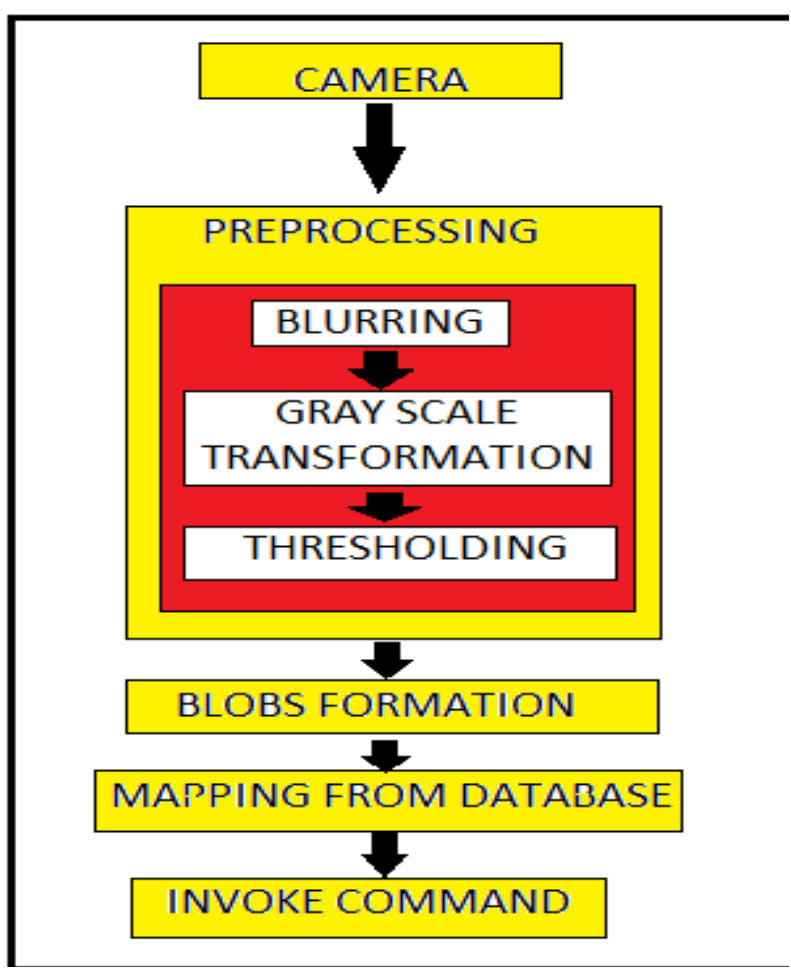

Fig 3. Hand Gesture Detection Module

\section{III.SHADER EFFECTS}

Shader effects is used to make 3-Dimentinal model of Augmented Reality more realistic. Due to such[5] kinds of effects the 3D model becomes realistic which will catch users eyes and leaves good impression about the product made of AR based GUIs. The tools used to impart shader properties on 3D models are Unity Engine and Vuforia. Shading is generally used to represent the effects and light on original object. Shader allows range of different rendering effect on the 3D virtual model.

There are two types of shaders.

A. Vertex Shader

In rendering process of virtual object vertex shaders is used to modifies the position of vertex, coordinates, texture. Basically vertex shader makes changes to the existing virtual object and does not create a new object.

\section{B. Pixel Shader}

The working of the pixel shader is to calculate the effect of individual pixel. It is easy to deal with the color of each pixel of the virtual object. It also help in dealing with the effect of shadow, lightening, transformation of the object.

In Unity Engine there is a shader module which is a computer program used to provide shading to the $3 \mathrm{D}$ object. Unity is a cross platform[6] used to develop games for various devices like PCs, mobile devices and consoles. Vuforia is a SDK that [7] enables creation of Augmented Reality application for mobiles devices.

\section{IV.OUR PROPOSED APPROACH}

The approach is utilising the features of Augmented Reality, mainly focussing on its ability to immerse user in a virtual environment. The aim of our approach is to deliver a platform to the user(which in this case is the student community) that would help them in learning various things with a higher efficiency. To further define the goal of this approach the intended platform would provide an experience to the user that would help them remember and recall things with greater ease. Few features including increased interactivity, openness to the virtual environment, gesture invocations are also planned to be a secondary part of the approach which would enhance the quality of experience the user would get.

The platform is Mobile Augmented Reality(MAR) application which can be developed by using Unity 3D engine and Vuforia. Once the MAR application is developed it is installed in the smartphones. After that the application can be used for learning. This application contain Augmented Reality based interfaces. 


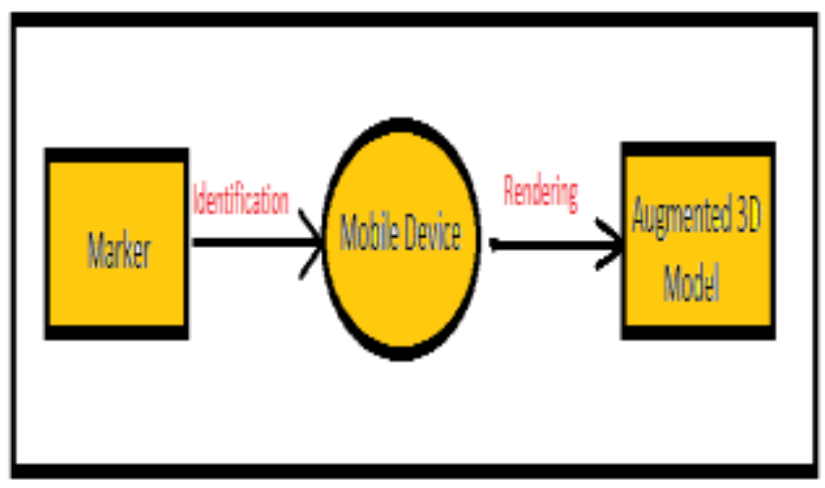

Fig. 4. Basic Mainframe of AR formation

\section{Conclusion}

In this paper we reviewed different learning based $A R$ interface models. All the AR models like Augmented Reality English Learning System and Picture Puzzle Education system they have a common aim to make learning things easy, interesting, fun and enhance the concentration of the user which include user involvement. The user can interact with the AR interface model via Hand Gestures. Shader effects which is a property used to make realistic 3D model of AR. Our approach is to develop a Mobile Augmented Reality (MAR) application through which user can interact with the application and have greater learning experience. The primary modules of the application contains Chemistry modules which shows different chemical reactions among different chemical components. The application contains another module of Solar System which gives information about it. The application helps the user to learn things of their interest in effective manner.

\section{REFERENCES}

[1] Min-Chai Hsieh, "Teachers and Student Perception Toward Augmented Reality Materials", Department of Information Management, Fortune Institute of Technology, Kaohsiung, Taiwan.

[2] Yeon-Jae Oh, Young-Sang Suh, Eung-Kon Kim, "Picture Puzzle Augmented Reality System for Infants Creativity", 978-1-46739991-3/16/ IEEE 2016.

[3] Purva Kedari, Puja Mahamulkar, Eram Khan, and Shalakha Kholi, "Augmented Reality using Hand Gesture Recognition System and its use in Virtual Dressing Room", International Journal of Innovation and Applied Studies ISSN 2028-9324 Vol. 10 No.1 Jan 2015,pp.95-100.

[4] Shivnarayan Rajappa and Gaurav Raj, "Application and Scope Analysis of Augmented Reality in Marketing Using Image Processing Technique”, 978-1-4673-8203-8/16/IEEE 2016.

[5] Hong-yi Pai. "An imitation of 3D projection mapping using augmented reality and shader effects", Department of Multimedia Design, National Formosa University.

[6] http://www.unity3d.com

[7] http://www.vuforia.com 